\title{
A NOTE ON SUBJUNCTIVE AND COUNTERFACTUAL IMPLICATION
}

\section{ROLF SCHOCK}

In January of 1962 , I was very surprised to hear that my paper on subjunctive and counterfactual implication had been published in volume II, number 4 (October, 1961) of this journal. Somehow, all proofs and communications from the editor were absorbed by the mailing process. I have not obtained the reprints, but the proofs are still on the road.

On opening the October issue of this journal, I was stunned; the printer had discarded the revised copy of the paper and had printed the original copy, errors and all, in its place.

Corrections which should have been made on the proofs are listed below.

\begin{tabular}{ll} 
Pages & \multicolumn{1}{c}{ Lines } \\
207 & 6 \\
& 7 \\
& $18-20$ \\
& \\
& \\
208 & $14,25-26$ \\
209 & 16
\end{tabular}

Write 'numbers' for 'number'.
Omit ' $\therefore$
Write ' $x$ satisfies one of the following conditions:

(1) $x$ is in the range of $s$

(2) there is an inner sequence $t$ of $s$

such that $x$ is in the range of $t$.' for these lines. Write 'ranges are' for 'range is'.

Omit ' $x$ is' and insert 'occurs in $\left\langle{ }^{\prime} \wedge\right.$ ' $\left.\nu\right\rangle$, there is no positive integer $n$ greater than $m$ such that the $n^{\text {th }}$ variable occurs in $\left\langle\wedge^{\prime}\right.$ ' $\left.v f\right\rangle$,' between 'variable' and 'and'. The improperness of the original clause was mentioned to me by Nino Cocchiarella.

9, 8 from bottom Omit line 8 and write ' $\because$ for 'or' in line 9.

2118

Interchange " $v_{2}$ '" and ' $v_{3}$ ',

9 from bottom Write 'ranges are' for 'range is'.

$212 \quad 1$

Insert 'for $d$ ' between 'assigner' and ' $x$ '.

Write ' $v$ ' for ' $w$ '.

2

Insert ',' between 'for $d$ ' and 'and $a$ '.

6 from bottom

Write 'relations' for 'relation'.

5 from bottom

Write 'could' for 'would'. 
Pages

Lines

$214 \quad 15,26$

217

219

\section{1}

8 from bottom 7

\section{Corrections}

Insert if is a formal empirically significant with respect to $i$ and $x$,' between ' $x$,' and 'and $p^{\prime}$.

Omit ' $\because$

Write 'for any $y$ in $d$ ' for 'for any $y$ in $a$ '.

Insert ' $d$ is a domain,' between 'interpreters,' and ' $i$ '.

It should be mentioned that

(1) the semantics of the paper can be made more elegant by relativizing semantic concepts to interpreters alone rather than to domains and interpreters;

(2) the definitions of satisfaction and truth for subjunctive and counterfactual conditionals can be widened by relativizing them to functions $p$ which assign deductive pairs to ordered pairs of formulas rather than to deductive pairs and using the weakest relations of subjunctive and counterfactual implication rather than the strongest ones; thus, we can say that the subjunctive conditional $\langle f$ ' $\boldsymbol{S}$ ' $g\rangle$ is satisfied in $d$ with respect $i$ and $x$ by $p$ and $m$ just in case the empty set subjunctively implies $g$ by $p$ (the pair $f, g$ ) on the basis of the set whose only member is $f$ in $d$ with respect to $i$ in the first sense for appropriate $d, i$, and $m$; and

(3) since, from the semantic point of view, subjunctive and counterfactual conditionals behave like formulas in which no variables occur free, it is actually more correct to understand ' $S$ ' and ' $C$ ' to be (like ' $N$ ', ' $E$ ', and ' $F$ ') $O$-place variable binders and so to write them in front of formulas instead of between them. 